\title{
Pattern Recognition in Narrative: Tracking Emotional Expression in Context
}

\author{
Fionn Murtagh ${ }^{1}$ and Adam Ganz ${ }^{2}$ \\ ${ }^{1}$ University of Derby, UK, and Goldsmiths University of London, UK \\ ${ }^{2}$ Royal Holloway, University of London, UK \\ Corresponding author: Fionn Murtagh, fmurtagheacm.org
}

\begin{abstract}
Using geometric data analysis, our objective is the analysis of narrative, with narrative of emotion being the focus in this work. The following two principles for analysis of emotion inform our work. Firstly, emotion is revealed not as a quality in its own right but rather through interaction. We study the 2-way relationship of Ilsa and Rick in the movie Casablanca, and the 3-way relationship of Emma, Charles and Rodolphe in the novel Madame Bovary. Secondly, emotion, that is expression of states of mind of subjects, is formed and evolves within the narrative that expresses external events and (personal, social, physical) context. In addition to the analysis methodology with key aspects that are innovative, the input data used is crucial. We use, firstly, dialogue, and secondly, broad and general description that incorporates dialogue. In a follow-on study, we apply our unsupervised narrative mapping to data streams with very low emotional expression. We map the narrative of Twitter streams. Thus we demonstrate map analysis of general narratives.
\end{abstract}

\section{Keywords}

narrative data mining, unsupervised classification, hierarchical classification, correspondence analysis, semantics, literature, filmscript, Twitter

\section{INTRODUCTION}

We establish an approach for mapping out narrative, with a primary focus on emotional attachment. Two extensively studied testbeds are used. Our methodology does not seek to isolate the qualities of interest. Rather, we map these qualities (i) through their interaction, and (ii) in context.

The new interdisciplinarity that we pursue here is between computational science and psychoanalysis. Longer term our aim is data-driven generative modelling. This is a different line of work relative to computational and psychological interdisciplinarity (Reisenzein et al. [2013]), largely using behavioural modelling. There are strong parallels here, in our view, with, respectively, unsupervised and supervised pattern recognition. Our approach is unsupervised, or data driven.

An interesting psychoanalytic view of the link between emotion and interaction is in Buchholz [2013]: "Emotions are a multifaceted object of interest. They should not be considered as producers of interaction. It might be more correct to reverse the order of causation: social interaction is the medium with the power to evoke and regulate human emotions." 
In brief, we carry out a metric embedding of the semantics of the discourse, and determine subplots of the narrative. From the metric embedding of the semantics we map the trajectory of emotional qualities, and emotional interactions of the characters. Also from the metric embedding of the semantics, we structure the continuity and change in the narrative using a hierarchy.

In section II, we present a short review of various strands of work in this area, that have lessons to be drawn. We indicate where, and why, our work differs.

Section III deals with our methodology of unsupervised pattern recognition.

Section IV relates to subplot analysis in the dialogue of Ilsa and Rick in the Casablanca movie. We ask: how is the overall dialogue of Ilsa and Rick to be understood in terms of subplots? Such subplots are found by us in decreasing order of importance or salience. They are determined in terms of factors, i.e. principal components or latent semantics. In section $\mathrm{V}$ we analyze emotion through emotional interaction. From the data, we find the major determinants of emotional interaction. This work is based on dialogue from the Casablanca movie. Since this has been very extensively analyzed, it provides us with an evaluation testbed.

Section VI is a study of the narrative and evolution of emotion from part of Flaubert's 19th century novel, Madame Bovary. Since we use text from this novel, with description as well as dialogue, this case study shows how well our methodology can handle general textual data.

In section VII we show the applicability of our unsupervised (or data-driven) methodology to very general narratives. We apply the principles of the methodology developed to Twitter streams. We map out the semantics through the Correspondence Analysis mapping of input frequency of occurrence data into the factor space. Next we consider sub-narratives from (statistically significant) segmentation of the Twitter stream. We locate, and then contrast, emotive words in the semantically-embedded structuring of the overall narrative.

\section{REVIEW OF DETERMINING AND TRACKING EMOTION IN VARIOUS APPLI- CATION DOMAINS}

We review in this section a number of approaches to determining and tracking emotional context in text and/or related data.

In subsection 2.1, lexical analysis based machine learning is used on social media data in order to label such data in terms of emotion or mood. In subsection 2.2 lexical analysis is used on novels in order to subtype them, and otherwise label them, in terms of emotional descriptors. The extrapolation of such an approach is proposed for characterizing the development of indications towards anti-social behaviour. Subsection 2.3 uses a hierarchy of mood states, that is manually created, and this is applied in a dynamic, evolving context.

\subsection{Supervised Lexical Analysis for Sentiment and Mood Analysis}

In Thelwall and Buckley [2013], positive or negative sentiment towards consumer products is the focus.

Two approaches are used - "mood setting" which characterizes social web texts on scales of +1 to +5 , and -1 to -5 , for selected sentiment words, with some context taken into account, such as questions, negations, exclamation marks, emoticons, and the use of the adverb "very". "Lexicon extension" (also referred to on page 3 of Thelwall and Buckley [2013] as lexical extension) seeks other additional words that are to a greater or lesser extent characteristic of alternative moods. 
In Paltoglou et al. [2013], rather than positive and negative scales of sentiment, "valence" and "arousal" are studied. In Généreux and Evans [2012], the terms "mood", "affective state" and "emotion" are identical. Schwartz's typology of motivational values is used, and these are further taken on two dimensions, positive/negative and passive/active. This study associated moral value terms with mood. Mood was defined by degree of the motivational values. Marked corpora were used.

Relative to the work described, which we can characterize as lexical and supervised, our work is unsupervised and is data driven, in particular insofar as uncontrolled term sets are determined through our pipeline processing and analysis. A further and crucial point of note is how the work described takes emotion as, very largely, mood, with a view towards prediction of a user's behaviour. Such behaviour can be purchase of a commodity, or an anti-social act.

\subsection{Emotion Tracking through Lexical Analysis}

In the emotion tracking project, Sutinen [2013], the approach taken is to determine terms and phrases that carry sentiment semantics, and then label them in degree of positivity or negativity. Examples of application areas are marketing and conflict prediction. The latter entails the search for "drastic changes in emotions towards a certain topic", Sutinen [2013]. (Multiple murder in a school shooting is at issue here, and in subsection 2.1 above, street rioting in the UK was at issue.)

It is noted in Kakkonen and Galić Kakkonen [2011] how sentiment analysis, as a new area of text analysis, has come to the fore in recent years. Emotion or affective content in novels is the focus, together with visualization. This work studies Gothic novels, that are "rich in dark and gloomy topics", hence negative emotions, and with sub-genres of terror and horror. A preestablished class hierarchy of nouns, verbs and adjectives is used. As an example, the "cruelty" class was associated with such words as "cruel", "pitilessness", "unkind". Sentiment-bearing words are scored, and these scores can be used to distinguish between the sub-genres noted, of terror and horror.

Where our work differs from Kakkonen and Galić Kakkonen [2011] is that we seek overall semantic characterization from data in an unsupervised, rather than supervised, way.

\subsection{Narrative of Emotion together with Narrative of Actions}

In this subsection, we review work focused on storyboarding or dramatization, together with plot unit summarization.

In addition to the storyline defined in Pizzi et al. [2007] as a sequence of actions (designed to address a task that the story defines), referred to as the plot, this work seeks to use "character" (Pizzi et al. [2007], section 1) also. In the plot, there is generation of action sequences. With character, the plot's actions are to be driven by intention and longer term motivations.

In an analysis of three chapters from Flaubert's 19th century novel, Madame Bovary, Cavazza et al. [2009], a top level polarity of feelings (states of mind) into "duty" and "pleasure" is used; and a set of five "emotional input" feelings, being combinations of "valence" and "arousal", that are determined in real time from the voice of a user interactively playing the role of Emma Bovary's lover, Rodolphe. These emotional input feelings are: NegativeActive, NegativePassive, Neutral, PositiveActive and PositivePassive.

In discussing other work, Cavazza et al. [2009] (in their section 6) note the objective being simulation and training, conferring believability on the virtual actors, while not at issue are 
narrative as such, or aesthetic aspects. It is acknowledged (in their section 7) that the emotional categories, for both state of feelings and expectations, are limited in number and genre-specific.

The modelling approach, originating in Schank and Abelson [1977], has led in the work of Lehnert [1981] to determining plot units from actors and actions, through use of "affect states" that characterize them, and weighted links that provide causal and cross-character values. Such a supervised approach is library-based (i.e. library of primitive plot units), with a taxonomy of affect states. Goyal et al. [2010] take such modelling considerably further, and include discussion of how to recognize affect states. In Goyal et al. [2010], 34 of Aesop's fables are used to exemplify this. Such a supervised modelling approach to the creation of plot unit structures is relevant in well-defined and well demarcated contexts.

\subsection{Dynamical Systems Modelling of Emotional States}

Rinaldi et al. [2013] model the Walt Disney film, "Beauty and the Beast", using state variables that represent feelings, and appeal functions. Emotions of partners in a relationship are modelled using ordinary differential equations. The dynamical system that ensues is then explored in terms of its system properties (such as chaotic behaviour). Parameter values are provided in analogy with the film content.

Khrennikov [2002, 2004] has developed and explored dynamical systems in p-adic (p prime) number systems. Cognitive process dynamics, represented as paths and trajectories in trees, imply that (see Khrennikov [2004] p. 138) a "pathway contributes to distinct psychological functions", and furthermore that "pathways going through reasoning-centres can go through some emotional centre. Thus reason participates in the creation of emotions and vice versa." Such dynamical systems work is not data-driven as in our approach.

\section{EMOTION ANALYSIS THROUGH UNSUPERVISED PATTERN RECOGNITION IN TEXTUAL DATA USAGE}

In this section, the principles of our methodology are discussed, together with various aspects relating to implementation.

For McKee [1999], text is the "sensory surface" of the underlying semantics. This is also what Benzécri [1979a,b, 1981] referred to as "letting the data speak", and "the model must follow the data and not the other way around". Our approach is one of (i) data encoding, (ii) measurement, including especially qualitative information through proxied expression of such information, (iii) pattern and trend determination and display, and thereafter (iv) model selection from the data.

\subsection{Euclidean Geometry for Mapping the Semantics, Tree Topology for Chronology of the Semantics}

Our starting point is the mapping of semantics into a visually-based representational space. This is accomplished by considering text (or other multimedia data) units crossed by the entities that comprise them and that serve to define them - words, in the case of text. Thus each text unit, e.g. a chapter of a novel, or scene of a filmscript, or one or some consecutive set of Twitter tweets, and so on, is contextually described by the frequencies of occurrence (including absence) of words, in the word set used. Similarly, each word has a vector of the text units in which it appears. Context, therefore, is part and parcel of our data from the start.

The choice of words in the word list includes - without loss of generality - setting all upper case to lower case and excluding punctuation. 
The dual spaces of text units and words are then mapped mathematically (and analytically using the eigenvalues and eigenvectors of these dual spaces, defined from the positive semidefinite matrix of their relationships) into a Euclidean space, termed a factor space (or principal component space, or latent semantic space). Planar projections of the factor spaces provide visualizations of the text units and words of the dual spaces. In Harcourt [2002] this is described as map analysis of qualitative observed data. These planar projections are based on decreasing informativeness of the factors, that are also referred to as space dimensions, or axes. Mathematically, the informativeness of the factors is defined by the moments of inertia of the clouds of points that are analysed. These are the dual clouds (mutually defining one another), the cloud of textual units employed, and the cloud of characterizing words. Through contribution to the inertia of a factor, we can read off the most contributing text units, and the most contributing words.

On the basis of the representation of the field of study, analysis of change over time, including continuity and change, next avails of a hierarchical mapping. We can require that the hierarchy be based on the chronology, or given sequence, of text units (e.g. dialogue elements, descriptive text segments). The algorithmic requirements to realise this (the pairwise agglomerative criterion that ensures a well-defined hierarchy, viz. with a monotonic sequence of agglomeration values: the complete linkage criterion) are described in Murtagh et al. [2009]. Typically the full dimensional field representation (i.e. factor space) is input to the hierarchy building algorithm.

For clustering, we are taking multiple scales into account through use of agglomerative hierarchical clustering. Secondly, rather than a typological analysis, what we want is to base the clustering on the chronology, or given sequence, of our text units. A further benefit of this approach is that, if required, we can statistically assess whether or not to carry out an agglomeration. This is based on a permutation test, in order to assess the statistical significance of a merger. This methodology is described in Bécue-Bertaut et al. [2014]. This furnishes a succession of clusters in our sequentially ordered set of text units. The succession of clusters is a segmentation of our time-line or overall narrative (expressed by the text units).

Sequence-constrained hierarchical clustering will be shown, below, to represent change in narrative direction. As noted, it takes scale into account. Relative to our narrative, this is scale of continuity and change. Using a basis in statistical significance testing, we can segment the narrative.

In summary, (i) our initial data is endowed with the $\chi^{2}$ metric (based on frequency counts, including zeros) in order to account for all semantic information; (ii) the dual (text, word) spaces are mapped into the factor space, and are endowed with the Euclidean metric (which can be said to be the natural, visual distance); (iii) the dual spaces, now in their Euclidean geometry, are mapped into an ultrametric topology. Ultrametric topology expresses hierarchy, or a tree structure.

\subsection{Analysis of Emotion: The Importance of Tool or Function Words}

In general we can distinguish between (i) tool words, form words, function words, empty words, grammar or grammatical words (examples: articles, prepositions, conjunctions, pronouns, and so on); and (ii) content words, full words. In Murtagh [2005], we cite many studies, using many languages, to support the following. Tool words are of importance for style analysis, and full words for content analysis. However style itself can be useful for describing context and relative properties. In particular ways, we can approach the analysis of content through tool 
words. Non-tool full words can be very specific, and therefore not at all helpful for comparative exploration of texts or text fragments.

Content words, with lemmatization and stemming, are important in supporting retrieval. Other forms of style and content can be relevant too, such as sentence length, patterns of punctuation usage, word length, use of the passive tense, etc. For us we make the following choice, given our aim in this work. The "texture" of the text is very important. Pennebaker [2012], and see also Moyer [2011], analyzed "junk" words (articles, pronouns, prepositions) and showed how they typified gender, and personal relationships (in the heading of Moyer [2011], "word usage predicts romantic attraction").

Thus we have a particular interest in tool or function words. In this context, there is no benefit to us in lemmatization and stemming. Note also that the very limited number of words used in dialogue precludes this.

In section VII, analysis of Twitter data, we employ a stoplist to remove function words. This is because there is far less concern for emotion-laden words.

\section{AN ANALYSIS OF EMOTIONAL INTERACTION IN THE MOVIE "CASABLANCA"}

In the movie Casablanca, to study emotion we look here specifically at the two main protagonists, or characters, Ilsa and Rick.

\subsection{Filmscript Text Data Used}

From the 77 scenes in Casablanca, we took the scenes where there was presence of both Ilsa and Rick. These were scene numbers 22, 26, 28, 30, 31, 43, 58, 59, 70, 75 and 77. We selected just the dialogue of the two, Ilsa and Rick, and in addition required that this dialogue be alternating. (There were some cases within a scene involving Ilsa and Rick where the dialogue of one of these involved other characters: such cases were not included, given our objective of focusing on direct Ilsa and Rick conversations.) Thus, dialogue elements were alternating between the two, Ilsa and Rick, with just a few instances of repeated utterances of the same person (Ilsa or Rick).

In all there were 150 dialogue utterances. The number of unique words in these utterances was 528, with: upper case set to lower case, punctuation set to blank, words constituted of $\geq 1$ contiguous letters. The number of these words that were used, including the speaker name for the utterance, was 2435. Next we required, to support the contrasting of utterances, that a word should be used a sufficient number of times. We required that each word be used in at least two different utterances, and that each word be used at least twice overall. This gave the total number of words retained as 261 .

The data input is a CSV file. We have made it available to the reader at www.narrativization.com. The first few dialogue utterances and the very final one are as follows, where we have Sequence Number (of the utterance), Name (Ilsa or Rick), and the Expression - the utterance. The dashes are as in the original script.

1, Rick: “- Hello, Ilsa.”

2, Ilsa: "Hello, Rick."

3, Ilsa: “- This is Mr. Laszlo.”

4, Rick: "How do you do?" 
5, Ilsa: "I wasn't sure you were the same. Let's see, the last time we met -"

6, Rick: “- It was La Belle Aurore.”

7, Ilsa: "How nice. You remembered. But of course, that was the day the Germans marched into Paris."

8, Rick: "Not an easy day to forget."

9, Ilsa: "No."

10, Rick: "I remember every detail. The Germans wore gray, you wore blue."

11, Ilsa: "Yes. I put that dress away. When the Germans march out, I'll wear it again."

150, Rick: "You better hurry, or you'll miss that plane."

\subsection{Semantic Mapping of the 150 Dialogue Utterances}

Our first analysis is frequency of occurrence cross-tabulations of the 150 utterances with the 261 word set. Figures 1 and 2 are separately displayed simply to avoid overcrowding. The factors (axes, components) are the principal axes of inertia of the cloud of weighted utterance points, and this overall cloud inertia is identical to the inertia of the cloud of weighted word points. The projections on this best fitting, principal factor, plane represent just $2.28 \%$ (first factor) plus $2.16 \%$ (second factor) of the inertia of the cloud of 150 utterances, or identically the inertia of the cloud of 261 words. This is typical for the sparse utterance/word data that is analyzed.

To focus our attention on the most influential utterances and words, the contribution to inertia of the factors is checked out. A threshold of 3 times the mean contribution was used here. This was a trade-off between decreasing inertia contributions, versus the need to restrict attention to a limited number of utterances and of words. Figures 1 and 2 show words and utterances that have inertia contributions that are greater than 3 times the mean (word or utterance, respectively) contribution. In Figure 2, utterances 17, 1, 2, 13, 68, 25, 147, 57 contributed strongly to the inertia and are on the negative side of the first factor. There were no single utterances contributing strongly to the positive side of the first factor. For the second factor, utterances 20, $145,28,42,36,52$, and also $1,2,13$, contributed strongly to the positive side of this factor. Contributing strongly to the negative side of factor 2 were utterances 72, 111.

While Figure 1 has the names "Ilsa" and "Rick" in the word set, i.e. among the 261 words, in Figure 2 we do the following. We use these names, i.e. the speaker of each of the utterances (coded for each utterance as 1 if the speaker, and as 0 if not the speaker), as supplementary attributes. That is, they are projected into the factor space subsequent to the determination of the factor space. They are passive attributes. It is interesting to note how these supplementary words have very close projections in the principal factor plane (Figure 2), similar to when they are used as words in the dialogue itself (Figure 1).

The utterances in Figure 2, that are the most important for this principal factor plane, are interesting as shown in the following list.

- Positive factor 1, positive factor 2 (nostalgic and endearing).

- 20, Rick: "Here's looking at you, kid."

145, Rick: "Here's looking at you, kid."

36, Rick, "You said it! Here's looking at you, kid."

120, Rick, "All right, I will. Here's looking at you, kid." 


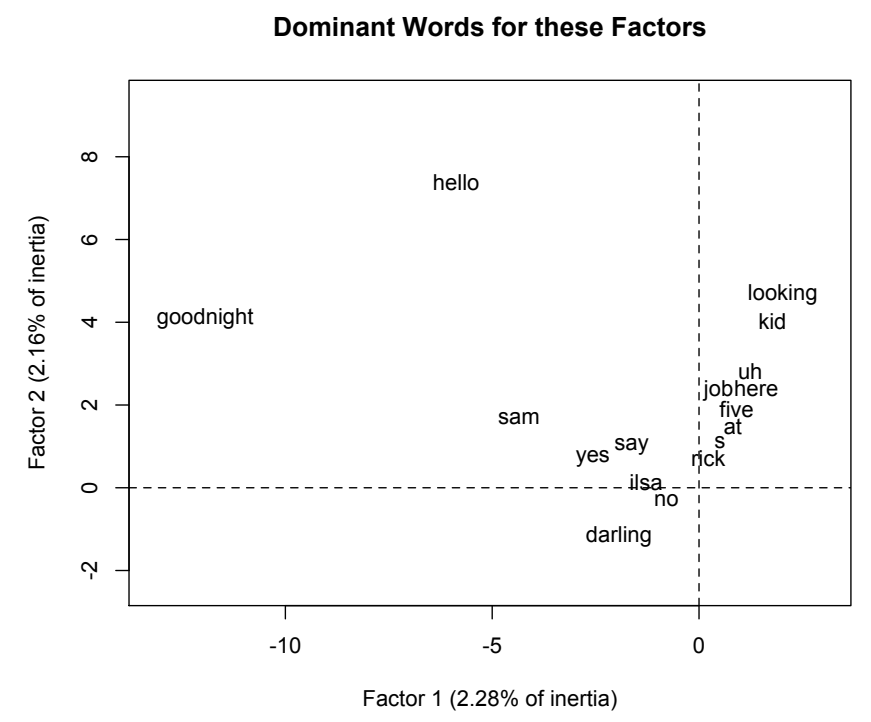

Figure 1: Principal plane of factors 1 and 2, resulting from the Correspondence Analysis, with the most contributing words (out of the 261 words) displayed. "s" is "is", resulting from, e.g., "here's". The words "Ilsa" and "Rick", in this display, are words used in the dialogue itself.

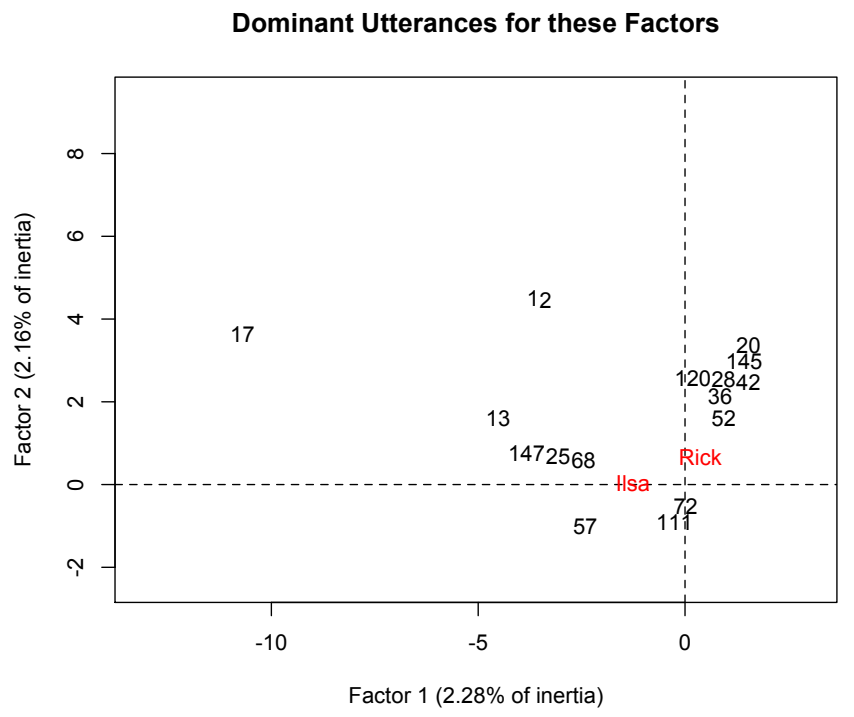

Figure 2: Principal plane of factors 1 and 2, resulting from the Correspondence Analysis, with the most contributing utterances (out of 150) displayed. The names "Ilsa" and "Rick", in this display, are supplementary attributes and are the speaker name, that is separate from the use of the person's name in a dialogue utterance. 
- 28, Rick: "Uh huh."

42, Rick: "Looking for a job."

- 52, Rick: "- The train for Marseilles leaves at five o'clock. I'll pick you up at your hotel at four-thirty."

- Negative factor 2, positive factor 1 (discursive and debating).

- 72, Ilsa, "Last night I saw what has happened to you. The Rick I knew in Paris, I could tell him. He'd understand. But the one who looked at me with such hatred... well, I'll be leaving Casablanca soon and we'll never see each other again. We knew very little about each other when we were in love in Paris. If we leave it that way, maybe we'll remember those days and not Casablanca, not last night."

111, Ilsa, "Oh, it wasn't my secret, Richard. Victor wanted it that way. Not even our closest friends knew about our marriage. That was his way of protecting me. I knew so much about his work, and if the Gestapo found out I was his wife it would be dangerous for me and for those working with me."

- 57, Ilsa, "Oh, darling!"

- Negative factor 2 (positive exchange, tending to being formal).

- 68, Ilsa, "Yes."

25, Ilsa, "Yes?"

147, Ilsa, "Yes."

- 13, Ilsa, "Say goodnight to Sam for me."

- 1, Rick, "- Hello, Ilsa."

2, Ilsa, "Hello, Rick."

17, Ilsa, "Goodnight."

Our semantic mapping has indicated the importance of the famous catch-phrase of the Casablanca movie script, "Here's looking at you, kid". We also note the importance of the one-word dialogue utterances, "Yes". We repeat a point made earlier: with such short utterances, we are truly studying the "texture" of the dialogue.

We note how important Sam, the piano player, is here in mediating the relationship of Ilsa and Rick (cf. upper left of the principal factor plane). The upper left quadrant is a somewhat formal and friendly space, referenced to the background context. In that sense, the Ilsa/Rick relationship is very much mediated by the ambient context. The upper right quadrant is, we could say informally, Rick's space. It is here where we find his celebrated, and repeated, phrase "Here's looking at you, kid." The lower left quadrant is, again informally, Ilsa's space, with a somewhat more amorous tendency.

We would characterize the factors as follows. Factor 1, negative, is dominated by an openly, positive, Ilsa. Factor 1, positive, is dominated by an endearing Rick. Factor 2, negative, is dominated by debating. Factor 2, positive, is dominated by comfortable exchange.

Were we to have a straightforwardly maturing relationship between Ilsa and Rick, then we might find the chronology of utterances in some simple pattern in the principal factor plane. We do not find such a pattern, because the relationship between these characters evolves through the film. The narrative and the subplots of the narrative are what we look at next.

\subsection{Semantic Analysis through Euclidean, Factor Space Embedding: Application to De- termining the Most Salient Subplots}

In order to examine the subplots that are interwoven into the overall narrative, we proceed as follows. The utterances, which are relevant for the emotional content as we have shown in the previous subsection, are not always suitable directly for studying the evolution of the narrative. 


\begin{tabular}{|l|rr|}
\hline Scene & \multicolumn{2}{|c|}{ Utterance } \\
& First in scene & Last in scene \\
\hline 22 & 1 & 17 \\
26 & 18 & 20 \\
28 & 21 & 31 \\
30 & 32 & 34 \\
31 & 35 & 60 \\
43 & 61 & 77 \\
58 & 78 & 107 \\
59 & 108 & 121 \\
70 & 122 & 128 \\
75 & 129 & 145 \\
77 & 146 & 150 \\
\hline
\end{tabular}

Table 1: The scene numbers (from the 77 scenes in the Casablanca script) with the first and last of the utterances that are from that scene. The utterances are (mostly consecutively) by either Ilsa or Rick, thus being dialogue expressions between these two characters.

(After all, there are a few utterances that just have "Yes" and "No". But in an emotional context, clearly they have their role.)

So, to study the evolution of the narrative, we use scenes. The scenes, and the dialogue utterances that come from them, are listed in Table 1. From the input data point of view, we aggregate the utterance data to provide the scene data. Therefore, we have up to now a 150 utterances $\times 261$ words table with 0 values whenever the word was not used in the utterance, and a value greater than 0 for the number of times the word was used in the utterance. (The largest such frequency of occurrence is 7 , and this is the case for "you" in utterance 95, and for "I" in utterance 113.) We aggregate rows 1 to 17 to form the new row of the scenes matrix, and so on (cf. Table 1). This gives us a frequency of occurrence, scenes $\times$ words, matrix of dimensions $11 \times 261$. This $11 \times 261$ matrix has 910 non-zero values and therefore has $31.7 \%$ occupancy. Its maximum value is 43 ("you" is used 43 times in one scene).

The factors, as the principal components, or latent variables, are the bearers of the information that underlies our data. They are given in order of decreasing importance. Therefore we will determine subplots in the overall narrative by looking at the factors, in succession. Since we can do that by displaying sets of planar projections, and also to economize on our discussion, we will discuss our findings by pairs of successive factors. We summarize our findings as follows, for the 11 scenes (and 261 words).

Factors 1 and 2: The most important scenes (in terms of contribution to the inertia defining the principal axes of inertia, or factors) are scene 22 and scene 31 . Scene 22 is where Ilsa and Rick meet again in Casablanca. Sam the piano-player and singer is present. At one point we have: "ILSA: Play it, Sam. Play 'As Time Goes By.'". Later there is this exchange: "RICK: Hello, Ilsa. ILSA: Hello, Rick." There is discussion of Germans in Paris in the past. The scene ends with Ilsa and Rick saying "Goodnight" to one another. In scene 31, there is a flashback to the past in Paris, with Sam there too, and ending with: "ILSA: Kiss me. Kiss me as if it were the last time.".

Therefore the positive quadrant for both factors is introductory and scene setting, based around Sam. Factor 2, negative half axis, refers to the past, in scene 31, and the positive half axis refers to the present, in particular scene 22. 
Factors 3 and 4: In the plane of factors 3 and 4, the preponderant scenes are as follows. Scenes 58 and 59, relate to Ilsa's husband Victor Laszlo ("he") getting transit papers to flee from Casablanca. Scenes 76 and 77 are at the airport where Rick sees off on the plane both Ilsa and Victor.

Whereas the plane of factors 1 and 2 relates to Ilsa and Rick meeting up again, the plane of factors 3 and 4 relates to their separation from one another.

Factors 5 and 6: In the plane of factors 5 and 6, scene 28 features, and this scene has Rick and Ilsa in a flashback to Paris, Rick in love with Ilsa stating that Victor Laszlo is dead, as she thought then. This plane also has as a preponderant scene, scene 77 which has Rick shooting dead Major Strasser, in self defence, and in order to allow Ilsa to escape from Casablanca with Victor.

If the planes of factors 1 and 2, and of factors 3 and 4, are respectively the beginning and the end of Ilsa's and Rick's renewed relationship, then the plane of factors 5 and 6 is a succinct, pithy summary, through the scenes displayed, of the overall narrative of this renewed relationship.

Factors 7 and 8: In the plane of factors 7 and 8, preponderant scene 28 is a Paris flashback, with a very short dialogue where Rick is in discovery mode about Ilsa (with "Who are you really?", and other questions to her). Then scene 43 is the market scene that we analyzed in Murtagh et al. [2009]. That latter scene is the time when Ilsa announces that she will leave Casablanca, and that Victor Laszlo has been her husband all along. Again therefore Rick is put (unwillingly) into discovery mode about Ilsa.

Factors 9 and 10: The plane of factors 9 and 10 counterposes scene 30, which in Paris points to Rick having to flee for safety, and Ilsa's concern for Rick's safety; with scene 70, where Rick indicates that he is helping both Victor and Ilsa flee for safety, and again Ilsa's concern for Victor is uppermost.

In summary we have the following.

1. The plane of factors 1 and 2: the beginning of the renewed meeting up of Ilsa and Rick in Casablanca, with the Paris background to this.

2. The plane of factors 3 and 4: the end of the renewed meeting up of Ilsa and Rick in Casablanca.

3. The plane of factors 5 and 6: a focus on what Rick did in the three-way relationship between Ilsa, Rick and Victor.

4. The plane of factors 7 and 8: subplot of Rick in discovery mode about Ilsa, actively back in Paris, and with Ilsa in the more active, revealing role, now in Casablanca. In the latter revelation, Victor enters decisively into their - Ilsa's, Rick's - relationship.

5. The plane of factors 9 and 10: subplot of Ilsa's concern for Rick, Ilsa's concern for Victor, and Rick's crucial support of Victor.

Our semantic analysis thus far has been firstly to determine emotional interaction between Ilsa and Rick, which was done through analysis of dialogue utterances (subsection 4.2). Then (this subsection 4.3) we determined subplots in the overall narrative. 


\section{CHRONOLOGY OF EMOTIONAL INTERACTION}

The following two principles for analysis of emotion inform our work.

- Emotion is revealed not as a quality in its own right but rather through interaction. Hence our interest in the direct interaction of the two characters, Ilsa and Rick.

- Emotion, that is expression of states of mind of subjects, is formed and evolves against the backdrop of external events and (personal, social, physical) context. Hence we traced out subplots (in subsection 4.3) because they define this context.

We now use the full factor, latent semantic embedding space. Hence no reduction in information is at issue, e.g. through a low dimensional semantic approximation.

\subsection{Evolution and Strength of Sentiment}

Our approach to tracking evolution of sentiment is unsupervised, i.e. data-driven. This differs from supervised approaches, that aim at classifying, in the sense of identifying, words as being of some label. For example, the classify_emotion program of Jurka [2012] in the R programming language, assesses text for labelling as one or more of these terms: "anger", "disgust", "fear", "joy", "sadness", "surprise". A trained naive Bayes classifier is used. Our interest in unsupervised analysis is in order to map the potentially very great complexity of what constitutes emotion. Ultimately, our interest is not in sentiment as mood but rather emotion as sentiments that express unconscious thought processes. See Murtagh [2014a].

Let us use particular words as proxies of strong emotional feeling. We recall that dialogue alone is at issue here (no metadata, nor any involvement by other characters), in scenes where Ilsa and Rick were exchanging successive utterances with each other. The word "kiss" was rare in the dialogue and therefore not retained for analysis. We used the words "darling" and "love". Figure 3 indicates that scene 31 is highest in terms of emotional attachment, as indicated by both of the sentiment-bearing words that we used. The last utterance recorded for Ilsa in this scene is: "Kiss me. Kiss me as if it were the last time." In scene 70, Rick assures Ilsa that he will help Victor Laszlo to escape, and thus there is less of a direct and immediate emotional attachment between the pair in that scene. The dénouement in the very last scene points to close emotional attachment, proxied by the word "love", as Ilsa and Victor fly away.

We see therefore how easy it is to track sentiment or other emotional states, through sentimentbearing or sentiment-expressing terms.

We additionally tracked, by scene, the terms "Rick" and "Ilsa" in the dialogue. We found these names as used in the dialogue to closely track the swings of movement ranging over low values in Figure 3 implying strong emotional attachment (e.g. in scene 31), and swings away from emotional attachment (e.g. in scene 70). We also found the differences, by scene, to be very small between Rick and Ilsa, again using these names from the dialogue. We did find differences, with Rick more imposing in scenes 22, 28, less so in 31, 58 and 70 (note that what we found was every second scene, with the exception of the very last), relative to Ilsa who was more imposing in all other scenes. However these differences between Rick and Ilsa were small. 


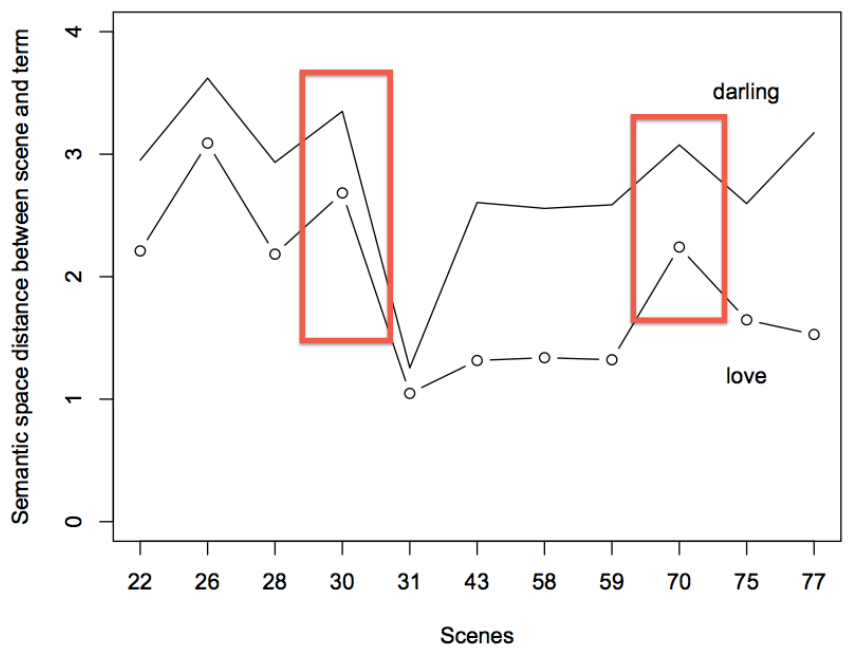

Figure 3: In the full dimensionality factor space, based on all interrelationships of scenes and words, we determined the distance between the word "darling" in this space, with each of the 11 scenes in this space. We did the same for the word "love". The semantic locations of these two words, relative to the semantic locations of scenes 30 and 70 are highlighted with boxes.

\subsection{Ultrametric Topology of Narrative}

The narrative of emotion, as expressed by the data we are using here, can be tracked in a parsimonious way by mapping the full dimensionality factor space data into a topology that respects the sequential or chronological order of the scenes. We use a sequence-constrained, or chronological, agglomerative hierarchical clustering algorithm.

In this particular case, we have found it beneficial to endow the correlation set, of each scene with all factors, with this ultrametric topology. Implementation-wise, this means that we use for each scene its set of correlations, i.e. cosines squared between the point and the axis (rather than the set of projections, or coordinates) in the factor space. The reason for doing this is to focus on relative orientation of the narrative, rather than on the evolving relative position of the narrative.

In Figure 4, we look at relatively large caesuras. Consider the partition with five clusters. A horizontal cut of the dendrogram at level 0.95 provides five clusters. The five cluster labels are, for the 11 scenes: $1,1,1,2,3,3,3,3,4,5,5$. The fourth and ninth scenes, viz. scene 30 and scene 70 , are singletons in this partition. There is discontinuity in the evolution of the narrative expressed by these scenes.

From Figure 3, the highlighted boxes refer to the cases of scenes 30 and 70. In Figure 3 these two scenes are a drifting apart of Rick and Ilsa. Such drifting apart is manifested there through somewhat greater distance of the semantic location of the terms "darling" or "love" from the overall semantic location of the scene. These relatively forceful discontinuities, given by the singleton clusters in the five-cluster partition in Figure 4, are indicative of the changes over scenes 28, 30, 31, and over scenes 59, 70, 75 .

If we look for the next discontinuity, determined by a partition with six clusters, and the next partition again with seven clusters, we find the crucial scene to be, respectively, 26 (the second 


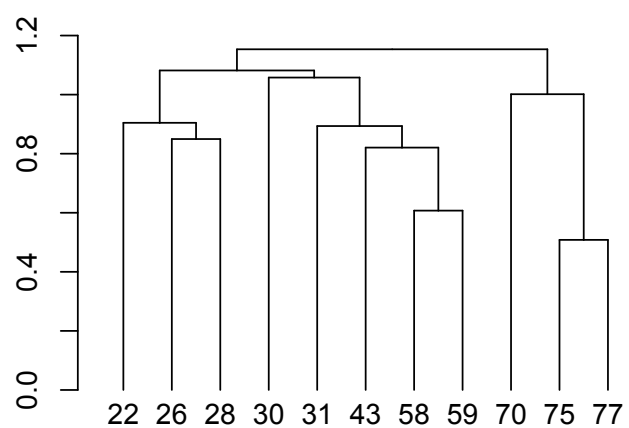

Figure 4: Hierarchical clustering, that is sequence constrained, of the 11 scenes used, i.e. scenes 22, 26, $28,30,31,43,58,5970,75,77$ (all with dialogue, and only dialogue, between Ilsa and Rick). Rather than projections on factors, here the correlations (or cosines of angles with factors) are used to directly capture orientation.

in sequence) and 31 (the fifth in sequence). Figure 3 provides an insightful perspective on what is happening to provide an explanation of these discontinuities.

In moving from scene 28 to 30 , the former here ends with a passionate kiss, and the latter is where Ilsa expresses her concern that Rick should flee Paris for his safety. In moving from scene 30 to 31, and then from 31 to 43, we have scene 31 where Ilsa and Rick are emotionally close, ending on Ilsa asking Rick to kiss her.

In moving from scene 59 to 70 , the former ends in emotionally proximity, while scene 70 is where Ilsa expresses her concern that Victor Laszlo needs to flee Casablanca for his safety. Then in moving from scene 70 to 75 , the latter details the start of the departure by plane of Ilsa and Victor.

In the summary of the two parts of Figure 4 that are most at issue in our discussion (namely, the transition between scenes 28,30,31, and the transition between scenes 59, 70, 75), we note affection, and non-affection is anxiety or apprehension. In line with other work reviewed in section II, we can easily label by scene the degree of affection, including emotional attachment, of the pair. This can be accomplished directly from Figure 3.

\section{NARRATIVE OF EMOTION IN FLAUBERT'S MADAME BOVARY}

The Casablanca work (i) analyzed strong emotional content, (i) it was based on dialogue, (iii) while it involved three characters (Ilsa, Victor, Rick), the emotional narrative was a narrative of attachment of two of these characters counterposed to anxiety or apprehension. In this Flaubert work we use the same methods (i) to analyze strong emotional content, (ii) based on dialogue and on extensive description provided by the novel, and (iii) we have a three-way interplay of the characters (Emma, Charles and Rodolphe). Because of the greater amount of data used, encompassing the dialogue, the emotional "signal" coming from this data is faint. We demonstrate the broad and general applicability of our methodology even with such faint emotional information. 


\subsection{Novel Text Data Used}

In particular because it was used in modelling mood in an interactive game context, Cavazza et al. [2009], we chose to look at the very emotional interplay between Emma Bovary and Rodolphe Boulanger in Chapters 9, 10, 11 and 12 of Flaubert's 19th century novel. With the four selected chapters of Madame Bovary, we have an initial task to address in regard to episodization or segmentation. Chafe [1994] in relating and establishing mappings between memory and story, or narrative, considered the following units: (i) memory expressed as a "disjointed chunk" of text; (ii) episode, expressed by a paragraph; (iii) thought, expressed by a sentence; and (iv) a focus, expressed by a phrase. For full generality of implementation, we adopted the following approach. Taking the four Madame Bovary chapters, the following preprocessing was carried out: upper case was set to lower case; punctuation was deleted; adjacent characters of length 1 or greater were words; and that furnished 3069 unique words, out of 14793 words used in total. We chose text units of 20 successive lines, giving rise to 22 successive text segments. This input data is available at address www.narrativization.com.

To have sufficient commonality of words, we required 5 occurrences of a word in a text segment, and furthermore at least a combined frequency of 5 presences of the word in all text segments. That resulted in the 22 text segments being cross-tabulated with 358 words. As previously, using frequency of occurrence values for the 22 text segments, crossed by the 358 words, we embedded word profiles, and text segment profiles in a Euclidean, factor, latent semantic space. (The term "profile" means the the frequency of occurrence vector, divided by the row or column total. The text segments comprise the rows, and the words comprise the columns.) From the full-dimensionality factor space, a chronological, sequence-respecting, hierarchical clustering was built. High contributing text segments were determined, that were more than 3 times the mean text segment contribution. High contributing words were similarly determined that were more than 6 times (due to the long tailed word frequency distribution) the mean word contribution.

\subsection{Mapping out the Chronology of the Emotional Triangle}

Figure 5 takes semantic location in the full dimensional factor space, and uses the text segment location in this same space as a reference point.

In Figure 5, with three personages, the following was used. We determine the distance squared to the text segment's semantic centre of gravity. The distance squared is calculated from the composite vector of Emma and Charles, her husband; and the composite vector of Emma and Rodolphe, her lover.

Mathematically, and hence algorithmically, this is as follows. Let $s_{k}$ be the $k$ th text segment, $f$ is the semantic vector of Emma, $m_{1}$ is the semantic vector of Rodolphe, and $m_{2}$ is the semantic vector of Charles. All these vectors are in the semantic or factor space. Figure 5 shows $\sqrt{d^{2}\left(s_{k}, f\right)+d^{2}\left(s_{k}, m_{1}\right)}$ and $\sqrt{d^{2}\left(s_{k}, f\right)+d^{2}\left(s_{k}, m_{2}\right)}$. Hence we have composition of the vectors of Emma and Rodolphe, and of Emma and Charles, in both cases referenced to, or centred in, the text segment.

Figure 5 presents an interesting perspective that can be considered relative to the original text. Rodolphe is emotionally scoring over Charles in text segment 1 , then again in $3,4,5$, 6 . In text segment 7, Emma is accosted by Captain Binet, giving her qualms of conscience. Charles regains emotional ground with Emma through Emma's father's letter in text segment 10, and Emma's attachment to her daughter, Berthe. Initially the surgery on Hippolyte in text segment 11 draws Emma close to Charles. By text segment 14 Emma is walking out on Charles following 


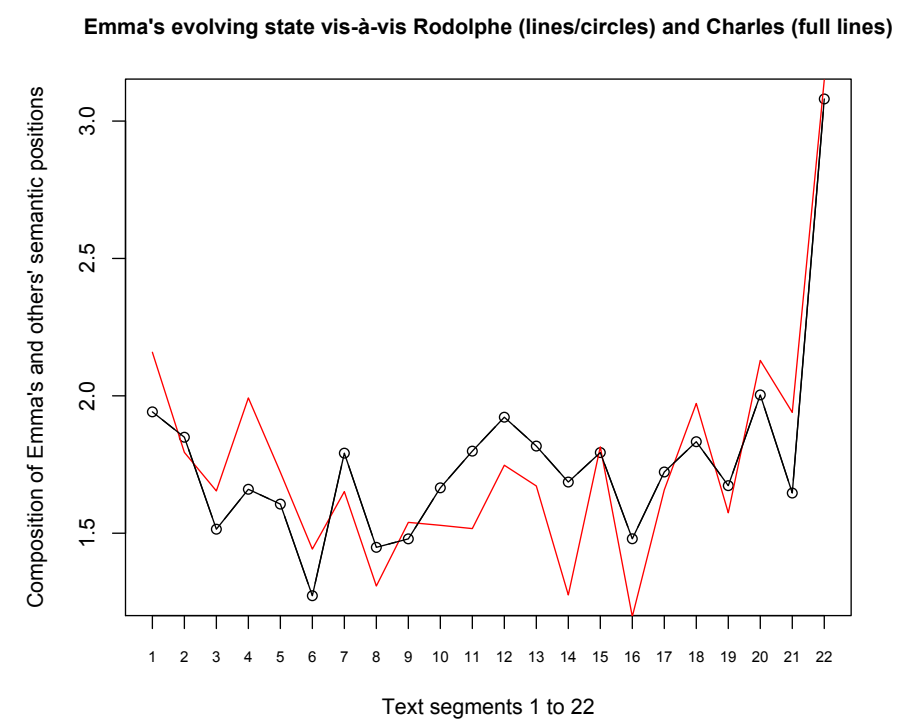

Figure 5: The relationship of Emma to Rodolphe (lines/circles, black) and to Charles (full line, red) are mapped out. The text segments encapsulate narrative chronology, that maps approximately into a time axis. Low or small values can be viewed as emotional attachment.

the botched surgery. Emma has total disdain for Charles in text segment 15. In text segment 16 Emma is buying gifts for Rodolphe in spite of potentially making Charles indebted. In text segments 17 and 18, Charles' mother is there, with a difficult mother-in-law relationship for Emma. Plans for running away ensue, with pangs of conscience for Emma, and in the final text segment there is Rodolphe refusing to himself to leave with Emma.

We recall that the text segments are somewhat crudely defined by us on the grounds of being easily defined. We find nonetheless that they provide interpretable and useful narrative flow. While Figure 5 provides a visualization of the interplay of the three characters, in Figure 6 we will visualize the relative strength of emotion.

\subsection{Tracking the Chronology of Emotional Sentiment}

Figure 6 displays the evolution of emotional sentiment, expressed by (or proxied by) the terms "kiss", "tenderness", and "happiness". We see that some text segments are more expressive of emotion than are other text segments.

\section{A GENERAL APPLICATION: EMOTIVE TERMS IN TWITTER NARRATIVES}

Following on from the two case studies that have been detailed, we address the following. Can our approach scale up in quantity of data? Can it be applied where there is no dominant emotional content? So we want to use our unsupervised narrative mapping approach in a general way. Our motivation is to consider data that mixes emotional and non-emotional content. In this section, we will be examining a word set of, in all, 22639 words.

Using the methodology that has been prototyped so far in this article, we now apply it, at scale, to social media. To demonstrate no limitations in regard to language, we use Twitter streams in English, French and German. To achieve our main objective of validating our methodology, news-related Twitter sources were taken. While very minimal in regard to emotional content as such, this data provides us with a useful validation framework. 


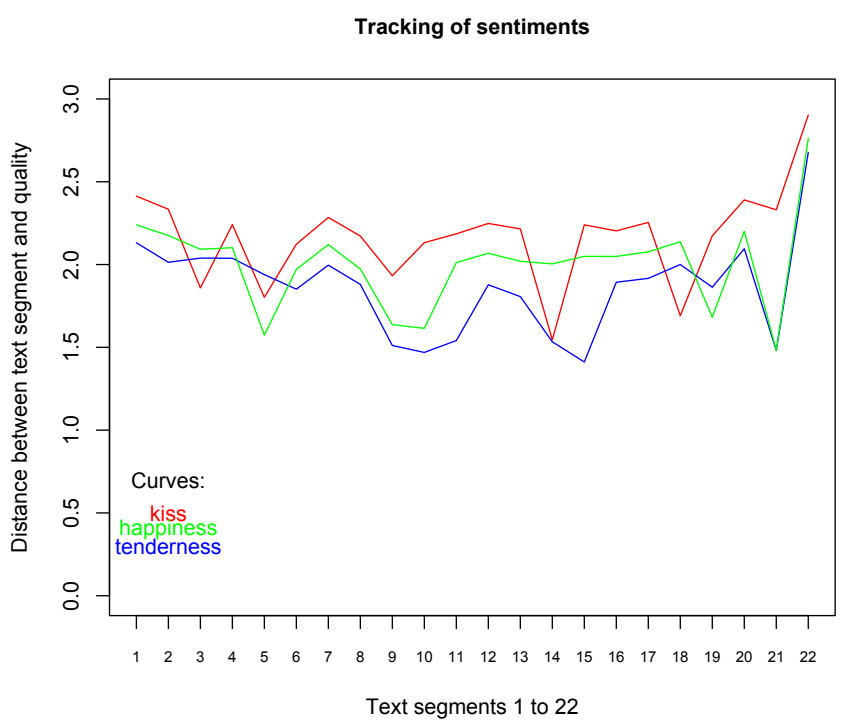

Figure 6: A low value of the emotion, expressed by the words "kiss", "happiness" and "tenderness", implies small distance to the text segment. These curves, "kiss", "happiness" and "tenderness" start on the upper left on the top, the middle, and the bottom, respectively. The chronology of sentiment tracks the closeness of these different sentimental terms relative to the narrative, represented by the text segment. Terms and text segments are vectors in the semantic, factorial space, and the full dimensionality of this space is used.

Validation here is to show how emotive terms (i) are baselined against the narrative flow, and (ii) can be contrasted, one to another, against this baselining.

We took the most recent 1000 tweets from the Twitter sources of the newspapers: New York Times, @nytimes; Le Monde, @lemondefr (in French); Guardian (UK), @guardian; Irish Times (Ireland), @ IrishTimes; Süddeutsche Zeitung, @ SZ (in German). We captured these tweet streams on 2014-10-17 (between 19:02 and 19:38 UTC). We have made this data available at www.narrativization.com. The 1000 tweets went back in time to between 2014-1008 and 2014-10-13 (for, respectively, the Irish Times and the Guardian; this shows a slightly greater degree of tweeting activity in the latter case).

For processing and matching convenience, we replaced the character "@" signifying a tweeter name by "XYZ", and the character "\#” signifying a topic by "ZYX". HTML ampersand was replaced by the word for "and" in the appropriate language. The apostrophe was replaced by a blank. All punctuation, and numeric characters, were deleted. All upper case was set to lower case. Stopwords, in the relevant language, and available from the SMART information retrieval system and accessed using the $\mathrm{R}$ tm, Text Mining Package, were removed. In Table 2 the number of words thus determined are listed for the 1000 selected tweets. Mostly, the frequency of occurrence of a word in a tweet was just 1 . The density of non-zero values in the tweets $x$ words data is reported on in Table 2.

Since our analytics are applied on the tweet stream, it is important that there be some commonality of words, rather than, say, a once and once only use of a word. From the experience of analyzing many types of text including Twitter, required thresholds are used of (i) word use in at least five tweets, and (ii) at least five uses of the word. In Table 2 there is note of the words retained that pass these thresholds. Thus the long tail of the word frequency distribution is cut. 


\begin{tabular}{lcccccc}
\hline Source & Tweets & Words & Density & Tweets(2) & Words(2) & Segments \\
\hline NY Times & 1000 & 4153 & 0.00183 & 846 & 247,247 & 31 \\
Le Monde & 1000 & 4428 & 0.00176 & 852 & 245,234 & 43 \\
Guardian & 1000 & 4637 & 0.00180 & 907 & 267,267 & 51 \\
Irish Times & 1000 & 3870 & 0.00207 & 917 & 275,269 & 50 \\
Südd. Z. & 1000 & 5551 & 0.00163 & 836 & 240,230 & 49 \\
\hline
\end{tabular}

Table 2: Summary of data properties. Tweets: initial number of tweets. Words: total number of words. Density: of tweets $\times$ words data. Tweets(2): non-empty tweets, given sufficient word occurrences. Words(2): number of sufficiently occurring words retained, followed by number of words for non-singleton tweet segments. Segments: tweet segments in tweet stream.

A consequence of this is that tweets may have all their (low frequency of occurrence) words removed. So Table 2 indicates the number of (now non-empty) tweets that were retained.

A metric embedding of the retained tweet cloud, and of the retained word cloud, was determined using Correspondence Analysis. Using the full dimensionality, in the Correspondence Analysis factor space endowed with the Euclidean distance, the retained tweet stream is segmented. For this, the complete link hierarchical agglomerative hierarchical clustering is used, with the following modification, as described in section 3.1. Rather than pairwise agglomerations until all the (contiguous) tweet clusters are in one overall cluster, here we statistically assess whether an agglomeration is to be permitted. Permitting an agglomeration is based on a permutation test of pairwise distances from randomization of groups. A $10 \%$ significance level is used. This results in a partition of the tweet sequence. We refer to these tweet clusters as segments in the stream of tweets. The numbers of segments found in the tweet streams are noted in Table 2. Singleton segments, or segments with a small number of tweets, may be of interest in terms of exceptionality but, here, our objective is to summarize the tweet flow by means of the successive tweet segments. For this reason, we restrict our attention to tweet segments that have more than one tweet. Doing this implies again that some words retained are no longer present. (That a segment has just one tweet is most consistent with relatively rare words being in use by that segment.)

These tweet flow segments define our Twitter narrative for us. They provide a particular summarization of the Twitter narrative. In a very high level sense, they respond to our need to periodize or break into episodes the narrative flow. (Indeed, Aristotle's Poetics, dating from c. $350 \mathrm{BC}$, refers to episodization, whereby story or narrative is developed through episodes that are elaborated on.)

Now we select emotive terms (notwithstanding the emotionally poor context, although this is helpful in limiting our choice of emotive terms). The semantically closest tweet segment to the term is noted. The tweet segment is noted using the time of the initial tweet, and the time of the last tweet. Universal time is used, UTC. The number of tweets in that semantically closest tweet segment are noted too. Our results are reported on in Table 3.

In all cases, the semantic distance to the tweet segment (using its semantic location) was plotted for the selected emotive terms. Figure 7 shows the outcome for one of the newspaper Twitter sources. Our emotive terms were selected by us for their positive or negative tendency, and it can be noted how this contrast is found in Figure 7. The (emotively) more positive leaning words have quite contrasting curves, i.e. distances to tweet segments, than is the case for the more negative leaning words. 


\begin{tabular}{llccc}
\hline $\begin{array}{l}\text { Tweet } \\
\text { Source }\end{array}$ & Emotive & $\begin{array}{c}\text { Tweet Segment } \\
\text { Start }\end{array}$ & $\begin{array}{c}\text { Tweet Segment } \\
\text { Finish }\end{array}$ & $\begin{array}{c}\text { Tweets in } \\
\text { Segment }\end{array}$ \\
\hline NY Times & hysteria & $2014-10-0907: 01: 26$ & $2014-10-0909: 49: 21$ & 13 \\
NY Times & threats & $2014-10-1516: 59: 11$ & $2014-10-15$ 21:00:18 & 37 \\
Le Monde & colère & $2014-10-1516: 58: 23$ & $2014-10-1516: 58: 23$ & 17 \\
Le Monde & espoirs & $2014-10-1203: 35: 10$ & $2014-10-1308: 13: 53$ & 36 \\
Guardian & worst & $2014-10-1409: 55: 23$ & $2014-10-1412: 28: 10$ & 60 \\
Guardian & threat & $2014-10-1518: 15: 32$ & $2014-10-1605: 35: 02$ & 10 \\
Irish Times & concerns & $2014-10-1506: 04: 05$ & $2014-10-1512: 06: 23$ & 42 \\
Irish Times & crisis & $2014-10-1405: 41: 34$ & $2014-10-1407: 17: 18$ & 16 \\
Südd. Z. & beste & $2014-10-0815: 45: 13$ & $2014-10-0818: 24: 14$ & 10 \\
Südd. Z. & erschreckende & $2014-10-0406: 37: 53$ & $2014-10-0414: 16: 59$ & 16 \\
\hline
\end{tabular}

Table 3: Emotive words ("colère" = anger; "espoirs" = hopes; "beste" = best; "erschreckende" = frightful). The Twitter narrative flow has been decomposed into narrative segments, see Table 2 . The closest segment to the emotive word is reported here. Semantic distance is used between the word and the tweet segment.

As we have shown, we locate semantically the selected terms, here emotive terms, in the narrative framework. The narrative framework is segmented, or if desired, hierarchically structured. Emotive terms like those used here are indicators of what we seek to study. In earlier sections, indicators were of amorous or other personal attachment. In the present section, dealing with news of national and international portent, indicators were of happier (or relaxed) versus sadder (or disturbing) periods. Finally, in this article, the narrative used has been literary story-telling, or cinematic script, or information dissemination.

Given the relevant data, further application of our work could be to the narrative of emotion,
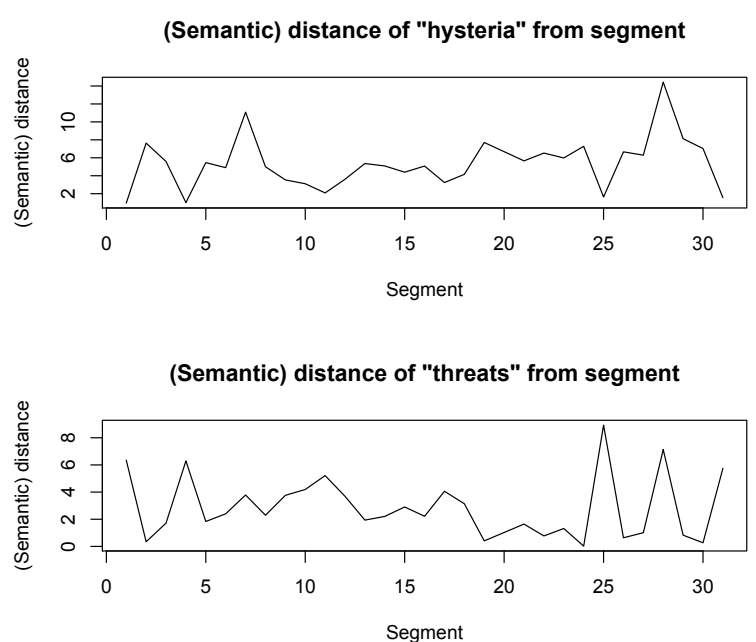

Figure 7: From 1000-tweet stream of the New York Times, anytimes. The Euclidean distances between the word and tweet segments 1 to 31 . All words and all tweet segments are embedded in the Correspondence Analysis factor space. This latent semantic space is endowed with the Euclidean metric and all points (words, tweet segments) are of equal mass. To aid in visualization, the distances are connected in a continuous curve. Small values of distance indicate a close semantic association of word and tweet segment. 
and action, for individuals or for groups of individuals.

\section{CONCLUSIONS}

Innovation in this work includes the determining of subplots of the overall narrative in section 4.3. In section 5.2 we develop further our approach for multiple scale, chronological representation of the narrative. In section 6.2 we have a new approach that displays the chronology of a 3-way emotional relationship. In section VII we open up our innovative methodology to the analysis of narrative in any codified form, expressed as text or otherwise.

We have exemplified our data driven approach to semantically mapping out narrative flow. By "semantics" we mean the entirety of given context. Any given thing is at the focal point of the totality of its interrelationships with all other things. Our mathematical and computational methodology allows us to structure narrative.

We applied this to (i) human dialogue, (ii) descriptive story, and (iii) streaming of information.

By semantically mapping out and structuring the narrative flow, we were able to semantically locate emotionally laden terms, or potentially emotive terms.

Potential applications of this work, that were noted in section II, include gamification, and social behaviour analysis and forensics. Other potential applications that we may note are new vistas in computational humanities, and computational psychoanalysis (cf. Murtagh [2014b]).

All data used in this article are available online, in the interest of reproducibility of results and of our findings. We have demonstrated the following in this work on literature and social media case studies. Our methodological high points are: (i) the potential of semantic mapping for theme tracing, and (ii) for theme subdivision; and also (iii) content-based segmenting of the flow of narrative.

\section{References}

M. Bécue-Bertaut, B. Kostov, A. Morin, and G. Naro. Rhetorical strategy in forensic speeches: multidimensional statistics-based methodology. Journal of Classification, 31:85-106, 2014.

J.P. Benzécri. Analyse des Données, Tome I: Correspondances. Dunod, Paris, 2 edition, 1979a.

J.P. Benzécri. Analyse des Données, Tome 2: Taxinomie. Dunod, Paris, 2 edition, 1979b.

J.P. Benzécri. Pratique de l'Analyse des Données, Tome 3: Linguistique et Lexicologie. Dunod, Paris, 1981.

M.B. Buchholz. Book review of J. Sidnell and T. Stivers, eds., The Handbook of Conversation Analysis, WileyBlackwell, 2013, and A. Peräkyländ and M.-L. Sorjonen, eds., Emotion in Interaction, Oxford University Press, 2012. Language and Psychoanalysis, 2(1):50-55, 2013.

M. Cavazza, D. Pizzi, F. Charles, T. Vogt, and E. André. Emotional input for character-based interactive storytelling. In AAMAS '09 Proceedings of 8th International Conference on Autonomous Agents and Multiagent Systems - Volume 1, pages 313-320, Richland, SC, USA, 2009. International Foundation for Autonomous Agents and Multiagent Systems.

W. Chafe. Discourse, Consciousness, and Time. The Flow and Displacement of Conscious Experience in Speaking and Writing. University of Chicago Press, 1994.

M. Généreux and R. Evans. Moods and moral values in blog posts. In WACAI 2012 - Workshop Affect, Compagnon Artificiel, Interaction, 2012. Grenoble, 6 pp., 15-16 November.

A. Goyal, E. Riloff, and H. Daumé III. Automatically producing plot unit representations for narrative text. In Proceedings of the 2010 Conference on Empirical Methods in Natural Language Processing (EMNLP 2010), pages 77-86, Stroudsburg, PA, 2010. Association for Computational Linguistics.

B.E. Harcourt. Measured interpretation: introducing the method of correspondence analysis to legal studies. University of Illinois Law Review, 2002(4):979-1018, 2002.

T.P. Jurka. R package sentiment, program classify_emotion, version 0.2. http://www.insider.org/packages/cran/sentiment/docs/classify_emotion, 2012. Accessed: 2015-04-27. 
T. Kakkonen and G. Galić Kakkonen. SentiProfiler: Creating comparable visual profiles of sentimental content in texts. In Proceedings of the Language Technologies for Digital Humanities and Cultural Heritage Workshop, 2011. Associated with the RANLP 2011 Conference, Hissar, Bulgaria, 8 pp.

A. Khrennikov. Classical and Quantum Mental Models and Freud's Theory of Unconscious Mind. Växjö University Press, 2002.

A. Khrennikov. Information Dynamics in Cognitive, Psychological, Social and Anomalous Phenomena. Kluwer, 2004.

W. Lehnert. Plot units and narrative summarization. Cognitive Science, 5(4):293-331, 1981.

R. McKee. Story: Substance, Structure, Style and the Principles of Screenwriting. Methuen, 1999.

M.W. Moyer. The language of love: word usage predicts romantic attraction. Scientific American, 2011. May/June.

F. Murtagh. Correspondence Analysis and Data Coding with Java and R. Chapman \& Hall/CRC Press, Boca Raton, FL, 2005.

F. Murtagh. Mathematical representations of Matte Blanco's bi-logic, based on metric space and ultrametric or hierarchical topology: towards practical application. Language and Psychoanalysis, 3(2):40-63, $2014 \mathrm{a}$.

F. Murtagh. Pattern recognition in mental processes: determining vestiges of the subconscious through ultrametric component analysis. In S. Patel, Y. Wang, W. Kinsner, D. Patel, G. Fariello, and L.A. Zadeh, editors, Proceedings ICCI*CC 2014, 2014 IEEE 13th International Conference on Cognitive Informatics and Cognitive Computing, pages 155-161, 2014b.

F. Murtagh, A. Ganz, and S. McKie. The structure of narrative: The case of film scripts. Pattern Recognition, 42: 302-312, 2009.

G. Paltoglou, M. Theunis, A. Kappas, and M. Thelwall. Predicting emotional responses to long informal text. IEEE Transactions on Affective Computing, 4(1):106-115, 2013.

J.W. Pennebaker. The Secret Life of Pronouns: What Our Words Say About Us. Bloomsbury Press, London, 2012.

D. Pizzi, M. Cavazza, and J.-L. Lugrin. Extending character-based storytelling with awareness and feelings. In AAMAS '07 Proceedings 6th International Joint Conference on Autonomous Agents and Multiagent Systems, ACM New York, NY, USA, 2007. Article No. 12.

R. Reisenzein, E. Hudlica, M. Dastani, J. Gratch, K. Hindriks, E. Lorini, and J.-J.Ch. Meyer. Computational modeling of emotion: toward improving the inter- and intradisciplinary exchange. IEEE Transactions on Affective Computing, 4(3):246-266, 2013.

S. Rinaldi, P. Landi, and F. Della Rossa. Small discoveries can have great consequences in love affairs: the case of Beauty and the Beast. International Journal of Bifurcation and Chaos, 23(11), 2013. 1330036.

R.C. Schank and R.P. Abelson. Scripts, Plans, Goals and Understanding. Lawrence Erlbaum, Mahwah, NJ, 1977.

E. Sutinen. Detecting and visualizing changes in emotions in texts. http://cs.joensuu.fi/ mmunez/emotion_detection/index.html, 2013. Academy of Finland research project 2010-2013, School of Computing, University of Eastern Finland. Accessed: 2015-04-27.

M. Thelwall and K. Buckley. Topic-based sentiment analysis for the social web: The role of mood and issue-related words. Journal of the American Society for Information Science and Technology, 64(8):1608-1617, 2013. 